\title{
INCOMPRESSIBLE FLUID FLOW COMPUTATION IN AN ARBITRARY TWO-DIMENSIONAL REGION ON NONSTAGGERED GRIDS
}

\author{
MIKHAIL CHUIKO \\ Institute of Mathematics, NAS of Belarus \\ 11 Surganov Str., 220072 Minsk, Belarus \\ E-mail: chuiko@im.bas-net.by \\ ANDREI LAPANIK \\ Belarussian State University \\ 4 Nezavisimosti Ave., 220050 Minsk, Belarus \\ E-mail: andrei@lapanik.name
}

\begin{abstract}
A numerical algorithm for solving the Navier-Stokes equations for incompressible viscous fluid in an arbitrary two-dimensional region on nonstaggered grids is presented. The idea of the transition to a general curvilinear coordinate system, transforming the physical region into a parametrical square is used. For the discrete solution an unconditional a priory estimate has been obtained. The results of the benchmark computations for a driven skewed cavity flow and the results of the fluid flow modeling in a cavity of an arbitrary shape are given.
\end{abstract}

2000 Mathematics Subject Classification: 65M06; 76D05.

Keywords: Navier-Stokes equations, finite difference method, curvilinear coordinate systems, nonstaggered grids.

\section{Introduction}

One approach to the numerical solution of partial differential equations in the regions of arbitrary shape is the introduction of general curvilinear coordinates transforming the initial region in the physical space into a parametrical square in the computational space of curvilinear coordinates [14]. The initial equations are transformed to new independent variables and are solved in the computational space on a rectangular difference grid making it possible to use the classical methods of the difference scheme theory.

The staggered $[6,8,12,16]$ and nonstaggered $[1,3,17]$ grids can be used for the numerical solution of the transformed Navier-Stokes equations in natural variables. The use of nonstaggered grids in the above papers supposes the interpolation of the velocity field to the cell faces.

In this paper difference schemes for the solution of the Navier-Stokes equations in an arbitrary region on nonstaggered grids based on the second order approximations in the 
grid nodes are used. In developing a computational algorithm, we have used an approximation of the diffusion operator with mixed derivatives, and for the elimination of the discrete solution oscillation we have introduced a pressure-containing regularizing term into the incompressibility condition. At the same time the convective difference operator has the property of cross-symmetry and the diffusion operator is self-adjoint and positive definite. For the discrete solution we have obtained an unconditional a priori estimate. The proposed algorithm for the computation of a viscous incompressible fluid flow in an arbitrary region is the generalization of the results obtained in [15] for the case of the rectangular region.

The potentialities of the method are illustrated by the test problem solutions $[2,7]$ and the results of modeling the fluid flow in a cavity with a curvilinear boundary.

\section{Problem formulation}

Consider a two-dimensional problem of determining the velocity components of an incompressible fluid flow as well as the pressure in an arbitrary two-dimensional region $\Omega$. The above parameters are determined from the incompressibility condition and the equation of motion, completed by the boundary and initial conditions

$$
\begin{gathered}
\frac{\partial \mathbf{v}}{\partial t}+C(\mathbf{v}) \mathbf{v}+\operatorname{grad} p-\frac{1}{\operatorname{Re}} \Delta \mathbf{v}=\mathbf{f}(x, y, t), \quad(x, y) \in \Omega, \quad 0<t \leqslant t_{0}, \\
\operatorname{div} \mathbf{v}=0, \quad(x, y) \in \Omega, \quad 0<t \leqslant t_{0}, \\
\mathbf{v}(x, y, t)=0, \quad(x, y) \in \partial \Omega, \quad 0<t \leqslant t_{0}, \\
\mathbf{v}(x, y, 0)=v_{0}(x, y), \quad(x, y) \in \Omega,
\end{gathered}
$$

where $C(\mathbf{v})$ is the convective operator, Re is the Reynolds number, $\mathbf{f}(x, t)$ is the source term, and $\Delta$ is the Laplace operator. Note that homogeneous boundary conditions of the first kind are used to simplify the presentation.

We use the skew-symmetric form of the convective term as the half-sum of convective terms in divergent and nondivergent forms [11]

$$
C(\mathbf{v}) \mathbf{v}=\frac{1}{2}((\mathbf{v} \cdot \operatorname{grad}) \mathbf{v}+\operatorname{div}(\mathbf{v} \mathbf{v})) .
$$

If the incompressibility condition (2) is satisfied, then the divergent, nondivergent and skewsymmetric forms of the convective term are equivalent.

For the unique determination of the pressure we use the additional condition

$$
\int_{\Omega} p(x, y, t) d x d y=0 .
$$

\section{Transition to a general curvilinear coordinate system}

One of the methods used to solve problems of mathematical physics in regions of arbitrary shape is the transition to a curvilinear coordinate system when the boundaries of the region coincide with the segments of the coordinate lines. The advantage of such an approach is a strict approximation of the transformed boundary conditions of any type without additional interpolation. 
We assume that there is a nonsingular one-to-one transformation $\xi=\xi(x, y), \eta=\eta(x, y)$, converting a physical region of an arbitrary shape $\Omega$ into the square region $\Omega_{\xi \eta}=\{(\xi, \eta)$, $0 \leqslant \xi \leqslant 1,0 \leqslant \eta \leqslant 1\}$ in the space of curvilinear coordinates $(\xi, \eta)$.

Problem (1)-(6) in the new variables is presented as follows:

$$
\begin{gathered}
\frac{\partial \mathbf{v}}{\partial t}+C_{\xi \eta}(\mathbf{v}) \mathbf{v}+\operatorname{grad}_{\xi \eta} p-\frac{1}{\operatorname{Re}} \Delta_{\xi \eta} \mathbf{v}=\overline{\mathbf{f}}(\theta, t), \quad \theta \in \Omega_{\xi \eta}, \quad 0<t \leqslant t_{0}, \\
\operatorname{div}_{\xi \eta} \mathbf{v}=0, \quad \theta \in \Omega_{\xi \eta}, \quad 0<t \leqslant t_{0}, \\
\mathbf{v}(\theta, t)=0, \quad \theta \in \partial \Omega_{\xi \eta}, \quad 0<t \leqslant t_{0}, \\
\mathbf{v}(\theta, 0)=\mathbf{v}_{0}(\theta), \quad \theta \in \Omega_{\xi \eta},
\end{gathered}
$$

where

$$
\begin{aligned}
\operatorname{div}_{\xi \eta} \mathbf{v} & =\frac{1}{J}\left[\frac{\partial}{\partial \xi}\left(\frac{\partial y}{\partial \eta} u\right)-\frac{\partial}{\partial \eta}\left(\frac{\partial y}{\partial \xi} u\right)-\frac{\partial}{\partial \xi}\left(\frac{\partial x}{\partial \eta} v\right)+\frac{\partial}{\partial \eta}\left(\frac{\partial x}{\partial \xi} v\right)\right], \\
\operatorname{grad}_{\xi \eta} p & =\frac{1}{J}\left(\frac{\partial y}{\partial \eta} \frac{\partial p}{\partial \xi}-\frac{\partial y}{\partial \xi} \frac{\partial p}{\partial \eta},-\frac{\partial x}{\partial \eta} \frac{\partial p}{\partial \xi}+\frac{\partial x}{\partial \xi} \frac{\partial p}{\partial \eta}\right) \\
C_{\xi \eta}(\mathbf{v}) \mathbf{v} & =\frac{1}{2}\left(\left(\mathbf{v} \cdot \operatorname{grad}_{\xi \eta}\right) \mathbf{v}+\operatorname{div}_{\xi \eta}(\mathbf{v v})\right) \\
\Delta_{\xi \eta} u & =\frac{1}{J}\left[\frac{\partial}{\partial \xi}\left(B_{11} \frac{\partial u}{\partial \xi}+B_{12} \frac{\partial u}{\partial \eta}\right)+\frac{\partial}{\partial \eta}\left(B_{12} \frac{\partial u}{\partial \xi}+B_{22} \frac{\partial u}{\partial \eta}\right)\right] \\
B_{11} & =\frac{g_{22}}{J}, \quad B_{12}=-\frac{g_{12}}{J}, \quad B_{22}=\frac{g_{11}}{J}, \\
g_{11} & =\left(\frac{\partial x}{\partial \xi}\right)^{2}+\left(\frac{\partial y}{\partial \xi}\right)^{2}, \quad g_{22}=\left(\frac{\partial x}{\partial \eta}\right)^{2}+\left(\frac{\partial y}{\partial \eta}\right)^{2}, \\
g_{12} & =\frac{\partial x}{\partial \xi} \frac{\partial x}{\partial \eta}+\frac{\partial y}{\partial \xi} \frac{\partial y}{\partial \eta}, \quad J=\frac{\partial x}{\partial \xi} \frac{\partial y}{\partial \eta}-\frac{\partial y}{\partial \xi} \frac{\partial x}{\partial \eta}
\end{aligned}
$$

Here $g_{11}, g_{12}, g_{22}$ are the metric coefficients, $J$ is the Jacobian of the inverse transformation $x=x(\xi, \eta), y=y(\xi, \eta)$.

It is easy to check that the equality

$$
B_{11} B_{22}-\left(B_{12}\right)^{2}=1, \quad \theta \in \Omega_{\xi \eta}
$$

is correct, so the corresponding quadratic form is positive definite [10]

$$
\sum_{n, m=1}^{2} B_{n m} \zeta_{n} \zeta_{m} \geqslant \nu \sum_{n=1}^{2}\left(\zeta_{n}\right)^{2}, \quad \theta \in \Omega_{\xi \eta}, \quad \nu>0 .
$$

Let $\mathcal{H}=L_{2, J}\left(\Omega_{\xi \eta}\right)$ be the Hilbert space of functions with the inner product

$$
(u, v)=\int_{\Omega_{\xi \eta}} u(\theta) v(\theta) J(\theta) d \theta
$$

and the corresponding norm $\|u\|^{2}=(u, u)$. We define the Hilbert space $\mathcal{H}^{2}=\mathcal{H} \oplus \mathcal{H}$ for the set of vectors $\mathbf{v}$ so that

$$
(\mathbf{u}, \mathbf{v})=\sum_{\alpha=1}^{2}\left(u_{\alpha}, v_{\alpha}\right)
$$


We define $\mathcal{H}_{*}^{2}$ as the subspace of $\mathcal{H}^{2}$ of the functions satisfying the incompressibility condition $\operatorname{div}_{\xi \eta} \mathbf{v}=0$.

To shorten the presentation, we write problem (7)-(10) in the following form:

$$
\begin{gathered}
\frac{d \mathbf{v}}{d t}+C_{\xi \eta}(\mathbf{v}) \mathbf{v}+P \mathbf{v}+N \mathbf{v}=\overline{\mathbf{f}}, \quad 0<t \leqslant t_{0}, \\
\mathbf{v}(\theta, 0)=\mathbf{v}_{0}(\theta), \quad \overline{\mathbf{f}}(\theta, t) \in \mathcal{H}^{2}, \quad \mathbf{v} \in \mathcal{H}_{*}^{2},
\end{gathered}
$$

where

$$
P \mathbf{v}=\operatorname{grad}_{\xi \eta} p, \quad N \mathbf{v}=-\frac{1}{\operatorname{Re}} \Delta_{\xi \eta} \mathbf{v}, \quad \mathbf{v} \in \mathcal{H}_{*}^{2} .
$$

The solution of problem (12) is sought on the subspace of functions from $\mathcal{H}_{*}^{2}$ vanishing on the boundary $\partial \Omega_{\xi \eta}$. At the same time the problem statement implicitly contains the boundary and incompressibility conditions, while notation (13) permits formal interpretation of the property of negative conjugation of the gradient and divergence operators as the property of skew-symmetry of the operator $P$.

Using the formulas of summation by parts and incompressibility condition (8), we prove that the operators $P$ and $C_{\xi \eta}(\mathbf{v})$ are skew-symmetric

$$
\begin{aligned}
(P \mathbf{v}, \mathbf{v}) & =\left(\operatorname{grad}_{\xi \eta} p, \mathbf{v}\right)=-\left(p, \operatorname{div}_{\xi \eta} \mathbf{v}\right)=0, \\
\left(C_{\xi \eta}(\mathbf{v}) \mathbf{v}, \mathbf{v}\right) & =0, \quad \mathbf{v} \in \mathcal{H}_{*}^{2},
\end{aligned}
$$

and, taking into account (11), the operator $N$ is positive definite

$$
(N \mathbf{v}, \mathbf{v})>0, \quad \mathbf{v} \in \mathcal{H}_{*}^{2} .
$$

Hence the following a priory estimate of the solution of nonlinear problem (12) is correct [11]:

$$
\|\mathbf{v}(\theta, t)\| \leqslant\left\|\mathbf{v}_{0}(\theta)\right\|+\int_{0}^{t_{0}}\|\overline{\mathbf{f}}(\theta, s)\| d s, \quad \theta \in \Omega_{\xi \eta} .
$$

In this case, the estimate ensures that the solution $\mathbf{v}$ is bounded.

\section{Difference approximation}

We introduce the uniform grid $\bar{\omega}=\omega \cup \partial \omega$ with steps $h_{\xi}$ and $h_{\eta}$ in the computational region $\Omega_{\xi \eta}$. Here $\omega$ is the set of internal nodes, $\partial \omega$ is the set of boundary nodes.

We approximate the metric coefficients and the Jacobian of the inverse transformation in the following way:

$$
\begin{aligned}
& \gamma_{11}=\left\{\begin{array}{ll}
\left(x_{\circ}\right)^{2}+\left(y_{\dot{\xi}}\right)^{2}, & (\xi, \eta) \in \omega, \\
\left(x_{\xi}\right)^{2}+\left(y_{\xi}\right)^{2}, & \xi=0, \\
\left(x_{\bar{\xi}}\right)^{2}+\left(y_{\bar{\xi}}\right)^{2}, & \xi=1,
\end{array} \quad \gamma_{22}= \begin{cases}\left(x_{\dot{\eta}}\right)^{2}+\left(y_{\dot{\eta}}\right)^{2}, & (\xi, \eta) \in \omega, \\
\left(x_{\eta}\right)^{2}+\left(y_{\eta}\right)^{2}, & \eta=0, \\
\left(x_{\bar{\eta}}\right)^{2}+\left(y_{\bar{\eta}}\right)^{2}, & \eta=1,\end{cases} \right.
\end{aligned}
$$

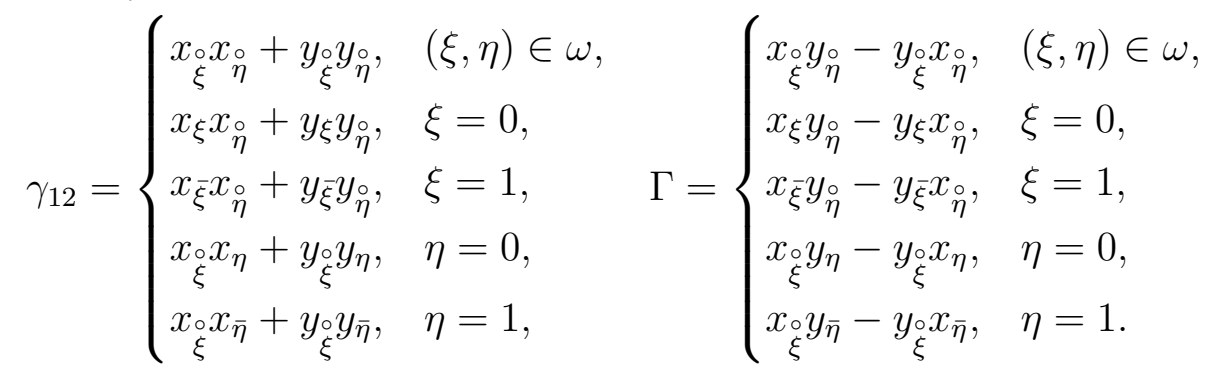


Here and further on we use the standard notation of the difference scheme theory from [9].

The coefficients $B_{11}, B_{12}, B_{22}$ are approximated in the form

$$
\beta_{11}=\frac{\gamma_{22}}{\Gamma}, \quad \beta_{12}=-\frac{\gamma_{12}}{\Gamma}, \quad \beta_{22}=\frac{\gamma_{11}}{\Gamma} .
$$

It is easy to check that the equality

$$
\beta_{11} \beta_{22}-\left(\beta_{12}\right)^{2}=1, \quad \theta \in \bar{\omega},
$$

is correct, so the corresponding quadratic form is positive definite

$$
\sum_{n, m=1}^{2} \beta_{n m} \zeta_{n} \zeta_{m} \geqslant \nu \sum_{n=1}^{2}\left(\zeta_{n}\right)^{2}, \quad \theta \in \bar{\omega}, \quad \nu>0 .
$$

Let $H$ be the finite-dimensional Hilbert space of grid functions with the inner product

$$
(q, z)=\sum_{\theta \in \omega} q(\theta) z(\theta) \Gamma(\theta) h_{\xi} h_{\eta}
$$

We define $H_{*}^{2}$ as the subspace of the functions from $H^{2}=H \oplus H$ vanishing on the boundary $\partial \omega$.

The gradient and divergence operators are approximated in such a way that the following equation is correct:

$$
\left(\operatorname{grad}_{h} p, \mathbf{w}\right)=-\left(p, \operatorname{div}_{h} \mathbf{w}\right)_{*}, \quad \mathbf{w} \in H_{*}^{2}
$$

where

$$
(q, z)_{*}=\sum_{\theta \in \omega} q(\theta) z(\theta) \Gamma(\theta) h_{\xi} h_{\eta}+\sum_{\theta \in \partial \omega} q(\theta) z(\theta) \Gamma(\theta) \frac{h_{\xi} h_{\eta}}{2} .
$$

Using second order approximations inside the computational region, the pressure gradient is approximated as follows:

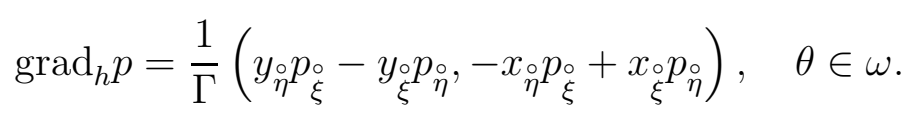

Taking into account the formulas of summation by parts and $\left.\mathbf{w}\right|_{\partial \omega}=0$, we write the approximation of the velocity divergence in the curvilinear coordinate system from equation (15) in the form

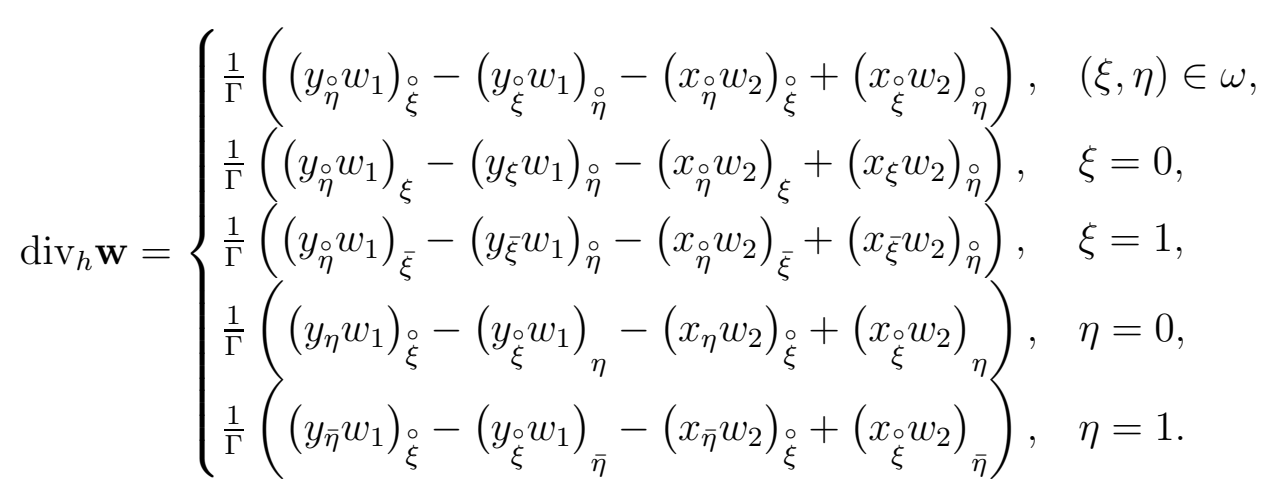

We consider the problem, differential with respect to time and differenced with respect to space, in accordance with problem (12)

$$
\begin{gathered}
\frac{d \mathbf{w}}{d t}+C_{h}(\mathbf{w}) \mathbf{w}+P_{h} \mathbf{w}+N_{h} \mathbf{w}=\overline{\mathbf{f}}, \quad 0<t \leqslant t_{0} \\
\mathbf{w}(\theta, 0)=\mathbf{v}_{0}(\theta)
\end{gathered}
$$


for smooth enough $\overline{\mathbf{f}}(\theta, t), \mathbf{v}_{0}(\theta)$. The solution of the problem is sought on a set of vectors w from the space $H_{*}^{2}$ of grid functions satisfying the difference incompressibility condition

$$
\operatorname{div}_{h} \mathbf{w}=0, \quad \theta \in \bar{\omega} .
$$

In problem (16), the difference operators are assigned as

$$
\begin{aligned}
P_{h} \mathbf{w}= & \operatorname{grad}_{h} p, \quad \mathbf{w} \in H_{*}^{2}, \\
C_{h}(\mathbf{w}) \mathbf{w}= & \frac{1}{2}\left(\left(\mathbf{w} \cdot \operatorname{grad}_{h}\right) \mathbf{w}+\operatorname{div}_{h}(\mathbf{w} \mathbf{w})\right), \\
N_{h} \mathbf{w}= & -\frac{1}{2 \operatorname{Re} \Gamma}\left[\left(\beta_{11} \mathbf{w}_{\bar{\xi}}\right)_{\xi}+\left(\beta_{11} \mathbf{w}_{\xi}\right)_{\bar{\xi}}+\left(\beta_{12} \mathbf{w}_{\bar{\eta}}\right)_{\xi}+\left(\beta_{12} \mathbf{w}_{\eta}\right)_{\bar{\xi}}\right. \\
& \left.+\left(\beta_{12} \mathbf{w}_{\bar{\xi}}\right)_{\eta}+\left(\beta_{12} \mathbf{w}_{\xi}\right)_{\bar{\eta}}+\left(\beta_{22} \mathbf{w}_{\bar{\eta}}\right)_{\eta}+\left(\beta_{22} \mathbf{w}_{\eta}\right)_{\bar{\eta}}\right] .
\end{aligned}
$$

From equations (15), (17) we get

$$
\left(P_{h} \mathbf{w}, \mathbf{w}\right)=\left(\operatorname{grad}_{h} p, \mathbf{w}\right)=-\left(p, \operatorname{div}_{h} \mathbf{w}\right)_{*}=0 .
$$

Using the analog of equation (15) for the velocity components

$$
\left(\left(\mathbf{w} \cdot \operatorname{grad}_{h}\right) w_{\alpha}, w_{\alpha}\right)=-\left(w_{\alpha}, \operatorname{div}_{h}\left(\mathbf{w} w_{\alpha}\right)\right),\left.\quad w_{\alpha}\right|_{\partial \omega}=0, \quad \alpha=1,2,
$$

we obtain

$$
\left(C_{h}(\mathbf{w}) \mathbf{w}, \mathbf{w}\right)=0
$$

Hence the operators $P_{h}$ and $C_{h}(\mathbf{w})$ are skew-symmetric.

The operator $N_{h}$ is self-adjoint and, according to (14), is positive definite [9]

$$
\left(N_{h} \mathbf{w}, \mathbf{w}\right)>0 .
$$

On a time-uniform grid with the time step $\tau$ we write the implicit scheme for problem (16) with a linearized convective term

$$
\begin{gathered}
\frac{\mathbf{w}^{n+1}-\mathbf{w}^{n}}{\tau}+C_{h}\left(\mathbf{w}^{n}\right) \mathbf{w}^{n+1}+N_{h} \mathbf{w}^{n+1}+P_{h} \mathbf{w}^{n+1}=\overline{\mathbf{f}}^{n} \\
\mathbf{w}(\theta, 0)=\mathbf{v}_{0}(\theta), \quad \mathbf{w} \in H_{*}^{2} .
\end{gathered}
$$

Finally, difference scheme (20) approximates problem (12) with order $O\left(|h|^{2}+\tau\right)$ on $\omega$ and $O(|h|)$ on $\partial \omega$.

\section{Computational algorithm with regularization}

Implementing scheme (20), we use the Douglas-Rachford splitting method [4]. As a result, the computational algorithm is divided into three stages.

At the first stage, we solve the following equation to get $\mathbf{w}^{n+1 / 2}$ :

$$
\frac{\mathbf{w}^{n+1 / 2}-\mathbf{w}^{n}}{\tau}+C_{h}\left(\mathbf{w}^{n}\right) \mathbf{w}^{n+1 / 2}+N_{h} \mathbf{w}^{n+1 / 2}+\operatorname{grad}_{h} p^{n}=\overline{\mathbf{f}}^{n} .
$$


At the second stage, we solve a discrete elliptical problem to calculate the pressure correction. For this purpose at the second fractional step we consider the equation

$$
\frac{\mathbf{w}^{n+1}-\mathbf{w}^{n+1 / 2}}{\tau}+\operatorname{grad}_{h}\left(p^{n+1}-p^{n}\right)=0 .
$$

Introducing notation $\delta p=p^{n+1}-p^{n}$, we rewrite (22) in the form

$$
\mathbf{w}^{n+1}=\mathbf{w}^{n+1 / 2}-\tau \operatorname{grad}_{h} \delta p, \quad \theta \in \omega .
$$

Finally, substituting (23) into (17), we get the following equation for the pressure correction $\delta p$

$$
\tilde{\Lambda} \delta p=\frac{1}{\tau} \operatorname{div}_{h} \mathbf{w}^{n+1 / 2}, \quad \theta \in \bar{\omega}
$$

where

$$
\begin{aligned}
& \tilde{\Lambda} p=\operatorname{div}_{h} \operatorname{grad}_{h} p=\tilde{\Lambda}_{\xi} p+\tilde{\Lambda}_{\eta} p,
\end{aligned}
$$

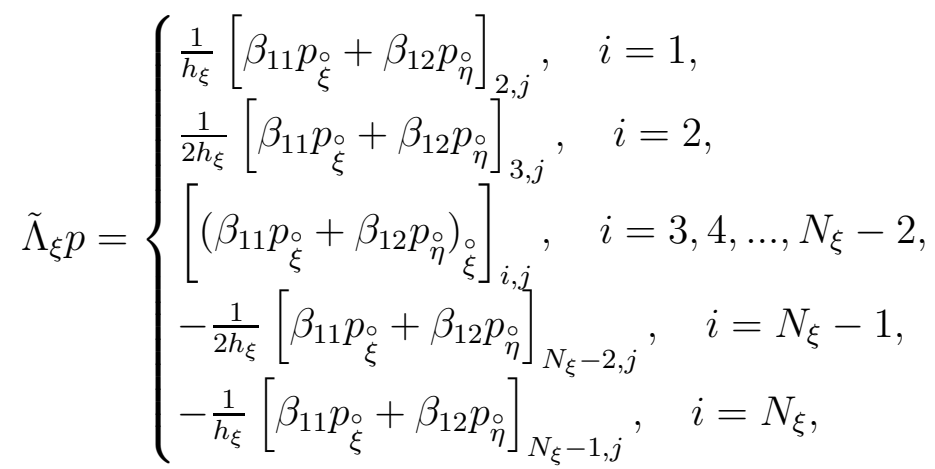

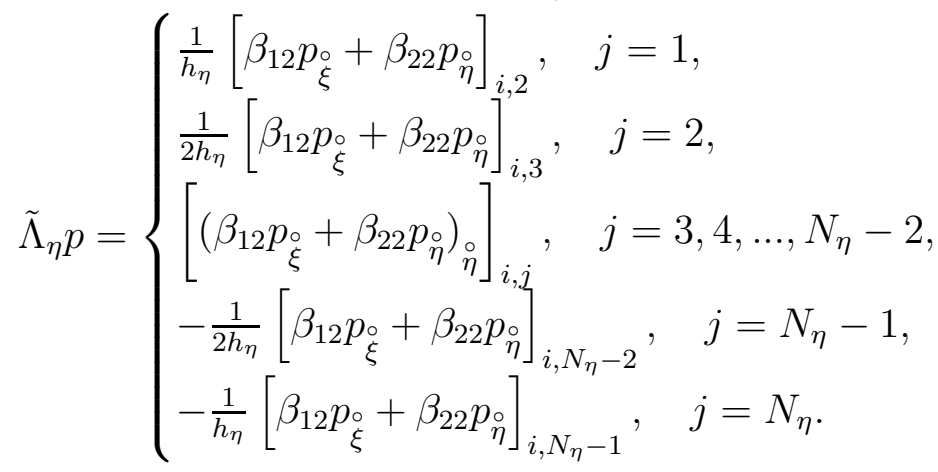

Note that the solution of problem (24) presupposes the solution of four discrete elliptical problems on nonintersecting sets of nodes of the grid $\bar{\omega}$, leading to oscillations of the discrete solution. To solve this problem, we suggest to introduce into the incompressibility condition a pressure-containing regularization term. Following the approach suggested in [15] for the case of a rectangular region, we demand that after introducing new terms

1. a positive definite and self-adjoint operator on a nonexpanded stencil should be used in the pressure correction problem;

2. additional terms do not make the order of approximation worse;

3. the general mass balance is not broken;

4. the operator $P_{h}$ should be nonnegative $\left(P_{h} \mathbf{w}, \mathbf{w}\right) \geqslant 0$ for obtaining an a priopi estimate of the discrete solution. 
To meet these demands, we consider the discrete analog of the incompressibility condition in the following form:

$$
\operatorname{div}_{h} \mathbf{w}^{n+1}=\tau(\Lambda-\tilde{\Lambda}) p^{n+1}, \quad \theta \in \bar{\omega}
$$

where

$$
\begin{aligned}
& \Lambda p=\Lambda_{\xi} p+\Lambda_{\eta} p, \\
& \left(\Lambda_{\xi} p\right)_{i, j}=\left\{\begin{array}{l}
\frac{1}{h_{\xi}}\left[\beta_{11} p_{\bar{\xi}}+\beta_{12} p_{\bar{\eta}}\right]_{2, j}, \quad i=1, \\
\frac{1}{2}\left[\frac{1}{h_{\xi}}\left(\beta_{11} p_{\xi}+\beta_{12} p_{\eta}\right)+\left(\beta_{11} p_{\bar{\xi}}+\beta_{12} p_{\bar{\eta}}\right)_{\xi}\right]_{i, j}, \quad i=2, \\
\frac{1}{2}\left[\left(\beta_{11} p_{\bar{\xi}}+\beta_{12} p_{\bar{\eta}}\right)_{\xi}+\left(\beta_{11} p_{\xi}+\beta_{12} p_{\eta}\right)_{\bar{\xi}}\right]_{i, j}, \quad i=3,4, \ldots, N_{\xi}-2, \\
\frac{1}{2}\left[-\frac{1}{h_{\xi}}\left(\beta_{11} p_{\bar{\xi}}+\beta_{12} p_{\bar{\eta}}\right)+\left(\beta_{11} p_{\xi}+\beta_{12} p_{\eta}\right)_{\bar{\xi}}\right]_{i, j}, \quad j=N_{\xi}-1, \\
-\frac{1}{h_{\xi}}\left[\beta_{11} p_{\xi}+\beta_{12} p_{\eta}\right]_{N_{\xi}-1, j}, \quad j=N_{\xi},
\end{array}\right. \\
& \left(\Lambda_{\eta} p\right)_{i, j}=\left\{\begin{array}{l}
\frac{1}{h_{\eta}}\left[\beta_{12} p_{\bar{\xi}}+\beta_{22} p_{\bar{\eta}}\right]_{i, 2}, \quad j=1, \\
\frac{1}{2}\left[\frac{1}{h_{\eta}}\left(\beta_{12} p_{\xi}+\beta_{22} p_{\eta}\right)+\left(\beta_{12} p_{\bar{\xi}}+\beta_{22} p_{\bar{\eta}}\right)_{\eta}\right]_{i, j}, \quad j=2, \\
\frac{1}{2}\left[\left(\beta_{12} p_{\bar{\xi}}+\beta_{22} p_{\bar{\eta}}\right)_{\eta}+\left(\beta_{12} p_{\xi}+\beta_{22} p_{\eta}\right)_{\bar{\eta}}\right]_{i, j}, \quad j=3,4, \ldots, N_{\eta}-2, \\
\frac{1}{2}\left[-\frac{1}{h_{\eta}}\left(\beta_{12} p_{\bar{\xi}}+\beta_{22} p_{\bar{\eta}}\right)+\left(\beta_{12} p_{\xi}+\beta_{22} p_{\eta}\right)_{\bar{\eta}}\right]_{i, j}, \quad i=N_{\eta}-1, \\
-\frac{1}{h_{\eta}}\left[\beta_{12} p_{\xi}+\beta_{22} p_{\eta}\right]_{N_{\eta}-1, j}, \quad i=N_{\eta} .
\end{array}\right.
\end{aligned}
$$

Substituting (23) into (25), we obtain the following discrete elliptical problem at the second stage of the computational algorithm

$$
\Lambda \delta p=\frac{1}{\tau} \operatorname{div}_{h} \mathbf{w}^{n+1 / 2}-(\Lambda-\tilde{\Lambda}) p^{n}, \quad \theta \in \bar{\omega} .
$$

At the third stage, the pressure and velocities are calculated on the next time level

$$
\begin{aligned}
p^{n+1} & =p^{n}+\delta p, \quad \theta \in \bar{\omega}, \\
\mathbf{w}^{n+1} & =\mathbf{w}^{n+1 / 2}-\tau \operatorname{grad}_{h} \delta p, \quad \theta \in \omega .
\end{aligned}
$$

We consider the properties of the operators $\Lambda$ and $\tilde{\Lambda}$ operating on $p \in H, \quad p(\theta) \neq$ $0, \quad \theta \in \partial \Omega$.

Taking into account the presentation of the operator $\Lambda$ in form (26), we consider $\Lambda_{\xi}$ separately:

$$
\begin{aligned}
\left(\Lambda_{\xi} q, z\right)_{*}=\frac{1}{2}[ & {\left[\beta_{11} q_{\bar{\xi}}+\beta_{12} q_{\bar{\eta}}\right]_{2, j} z_{1, j} h_{\eta}+\left[\beta_{11} q_{\xi}+\beta_{12} q_{\eta}\right]_{2, j} z_{2, j} h_{\eta} } \\
& +\sum_{j=2}^{N_{\eta}-1} \sum_{i=2}^{N_{\xi}-2}\left[\left(\beta_{11} q_{\bar{\xi}}+\beta_{12} q_{\bar{\eta}}\right)_{\xi}\right]_{i, j} z_{i, j} h_{\xi} h_{\eta}+\sum_{j=2}^{N_{\eta}-1} \sum_{i=3}^{N_{\xi}-1}\left[\left(\beta_{11} q_{\xi}+\beta_{12} q_{\eta}\right)_{\bar{\xi}}\right]_{i, j} z_{i, j} h_{\xi} h_{\eta} \\
& \left.-\left[\beta_{11} q_{\bar{\xi}}+\beta_{12} q_{\bar{\eta}}\right]_{N_{\xi}-1, j} z_{N_{\xi}-1, j} h_{\eta}-\left[\beta_{11} q_{\xi}+\beta_{12} q_{\eta}\right]_{N_{\xi}-1, j} z_{N_{\xi}, j} h_{\eta}\right]
\end{aligned}
$$

and using the formulas of summation by parts, we obtain

$$
\left(\Lambda_{\xi} q, z\right)_{*}=-\frac{1}{2} \sum_{j=2}^{N_{\eta}-1} \sum_{i=2}^{N_{\xi}-1}\left[\left(\beta_{11} q_{\bar{\xi}}+\beta_{12} q_{\bar{\eta}}\right) z_{\bar{\xi}}+\left(\beta_{11} q_{\xi}+\beta_{12} q_{\eta}\right) z_{\xi}\right]_{i, j} h_{\xi} h_{\eta} .
$$


The similar equation can be written for $\Lambda_{\eta}$ :

$$
\left(\Lambda_{\eta} q, z\right)_{*}=-\frac{1}{2} \sum_{i=2}^{N_{\xi}-1} \sum_{j=2}^{N_{\eta}-1}\left[\left(\beta_{12} q_{\bar{\xi}}+\beta_{22} q_{\bar{\eta}}\right) z_{\bar{\eta}}+\left(\beta_{12} q_{\xi}+\beta_{22} q_{\eta}\right) z_{\eta}\right]_{i, j} h_{\xi} h_{\eta} .
$$

Finally, the following equation for $\Lambda$ is correct:

$$
\begin{aligned}
(\Lambda q, z)_{*}= & -\frac{1}{2} \sum_{i=2}^{N_{\xi}-1} \sum_{j=2}^{N_{\eta}-1}\left[\beta_{11} q_{\bar{\xi}} z_{\bar{\xi}}+2 \beta_{12} q_{\bar{\xi}} z_{\bar{\eta}}+\beta_{22} q_{\bar{\eta}} z_{\bar{\eta}}\right. \\
& \left.+\beta_{11} q_{\xi} z_{\xi}+2 \beta_{12} q_{\xi} z_{\eta}+\beta_{22} q_{\eta} z_{\eta}\right]_{i, j} h_{\xi} h_{\eta} .
\end{aligned}
$$

In the same way for $\tilde{\Lambda}$ we get

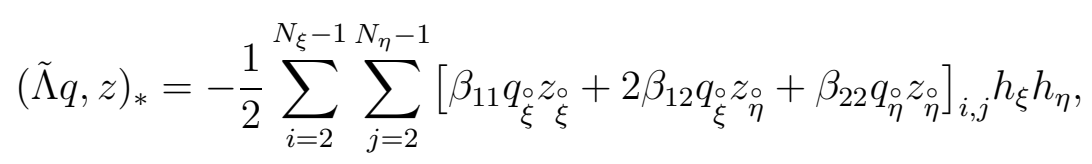

hence the operators $\Lambda$ and $\tilde{\Lambda}$ are self-adjoint

$$
(\Lambda q, z)_{*}=(q, \Lambda z)_{*}, \quad(\tilde{\Lambda} q, z)_{*}=(q, \tilde{\Lambda} z)_{*} .
$$

Using (14), we prove that the operators $\Lambda$ and $\tilde{\Lambda}$ are positive definite

$$
(-\Lambda p, p)_{*} \geqslant 0, \quad(-\tilde{\Lambda} p, p)_{*} \geqslant 0 .
$$

Now we consider the properties of the regularization term. We can prove that

$$
((\Lambda-\tilde{\Lambda}) p, p)_{*}=-\sum_{i=2}^{N_{\xi}-1} \sum_{j=2}^{N_{\eta}-1}\left[\beta_{11}\left(\frac{p_{\xi \bar{\xi}}}{2}\right)^{2}+2 \beta_{12} \frac{p_{\xi \bar{\xi}}}{2} \frac{p_{\eta \bar{\eta}}}{2}+\beta_{22}\left(\frac{p_{\eta \bar{\eta}}}{2}\right)^{2}\right]_{i, j} h_{\xi} h_{\eta} .
$$

Taking into account (14), the following inequality is correct:

$$
-((\Lambda-\tilde{\Lambda}) p, p)_{*} \geqslant 0
$$

hence, we prove that the operator $P_{h}$ is positive definite

$$
\left(P_{h} \mathbf{w}, \mathbf{w}\right)=\left(\operatorname{grad}_{h} p, \mathbf{w}\right)=-\left(p, \operatorname{div}_{h} \mathbf{w}\right)_{*}=-(p, \tau(\Lambda-\tilde{\Lambda}) p)_{*} \geqslant 0 .
$$

On the set of functions with the normal derivative vanishing on a boundary the operator $\Lambda$ approximates the Laplace operator with the first order and the operator $\tilde{\Lambda}$ — with the second order. This restriction comes with the need of obtaining equation (28) and estimates (29), (30) consequently.

Taking into account that the operators $\Lambda$ and $\tilde{\Lambda}$ are self-adjoint, we write

$$
(\tau(\Lambda-\tilde{\Lambda}) p, 1)_{*}=(p, \tau(\Lambda-\tilde{\Lambda}) 1)_{*}=0,
$$

thus, the introduction of the additional term into the incompressibility condition does not brake the difference analog of the integral mass conservation law. The discrete analog of the solvability condition of problem (27) in the form

$$
\left(\frac{1}{\tau} \operatorname{div}_{h} \mathbf{w}-(\Lambda-\tilde{\Lambda}) p, 1\right)_{*}=0, \quad \mathbf{w} \in H_{*}^{2}, \quad p \in H
$$

follows from (32) and (15). 


\section{Discrete solution estimate}

To obtain an a priory estimate of the discrete solution following [15], we write equations (21) and (22) in the form

$$
\begin{aligned}
\left(E+\tau A_{1}\right) \mathbf{w}^{n+1 / 2} & =\left(E-\tau A_{2}\right) \mathbf{w}^{n}+\tau \overline{\mathbf{f}}^{n}, \\
\left(E+\tau A_{2}\right) \mathbf{w}^{n+1} & =\mathbf{w}^{n+1 / 2}+\tau A_{2} \mathbf{w}^{n},
\end{aligned}
$$

where

$$
A_{1}=C_{h}(\mathbf{w})+N_{h}, \quad A_{2}=P_{h} .
$$

Taking into account equations (18), (19) and (31), we get

$$
\left(A_{1} \mathbf{w}, \mathbf{w}\right)>0, \quad\left(A_{2} \mathbf{w}, \mathbf{w}\right) \geqslant 0, \quad \mathbf{w} \in H_{*}^{2} .
$$

We consider the following intermediate equation:

$$
\left(E+\tau A_{2}\right) \mathbf{w}^{n+1}=\frac{1}{2}\left(E+\tau A_{2}\right) \mathbf{w}^{n}+\frac{1}{2}\left(E-\tau A_{1}\right) \mathbf{w}^{n+1 / 2}+\frac{\tau}{2} \overline{\mathbf{f}}^{n},
$$

as the result of the composition of equation (33) and equation (34) multiplied by two.

Multiplying it scalarly by $\left(E+\tau A_{2}\right) \mathbf{w}^{n+1}$ and using the Shwarz inequality for the right hand side estimate, we obtain

$$
\left\|\left(E+\tau A_{2}\right) \mathbf{w}^{n+1}\right\| \leqslant \frac{1}{2}\left\|\left(E+\tau A_{2}\right) \mathbf{w}^{n}\right\|+\frac{1}{2}\left\|\left(E-\tau A_{1}\right) \mathbf{w}^{n+1 / 2}\right\|+\frac{\tau}{2}\left\|\overline{\mathbf{f}}^{n}\right\| .
$$

In the same way for (33) we get

$$
\left\|\left(E+\tau A_{1}\right) \mathbf{w}^{n+1 / 2}\right\| \leqslant\left\|\left(E-\tau A_{2}\right) \mathbf{w}^{n}\right\|+\tau\left\|\overline{\mathbf{f}}^{n}\right\| .
$$

Using the following inequality for any operator $B \geqslant 0$ :

$$
\|(E-B) y\| \leqslant\|(E+B) y\|,
$$

and inequality (36), we obtain the estimate of the second term of the right hand side of inequality (35)

$$
\begin{aligned}
\left\|\left(E-\tau A_{1}\right) \mathbf{w}^{n+1 / 2}\right\| & \leqslant\left\|\left(E+\tau A_{1}\right) \mathbf{w}^{n+1 / 2}\right\| \leqslant\left\|\left(E-\tau A_{2}\right) \mathbf{w}^{n}\right\|+\tau\left\|\overline{\mathbf{f}}^{n}\right\| \\
& \leqslant\left\|\left(E+\tau A_{2}\right) \mathbf{w}^{n}\right\|+\tau\left\|\overline{\mathbf{f}}^{n}\right\| .
\end{aligned}
$$

Finally, the following estimate is correct:

$$
\left\|\left(E+\tau A_{2}\right) \mathbf{w}^{n+1}\right\| \leqslant\left\|\left(E+\tau A_{2}\right) \mathbf{w}^{n}\right\|+\tau\left\|\overline{\mathbf{f}}^{n}\right\|,
$$

hence

$$
\left\|\left(E+\tau A_{2}\right) \mathbf{w}^{n+1}\right\| \leqslant\left\|\left(E+\tau A_{2}\right) \mathbf{w}^{0}\right\|+\sum_{k=0}^{n} \tau\left\|\overline{\mathbf{f}}^{k}\right\| .
$$

Taking into account the equality

$$
\begin{aligned}
\left\|\left(E+\tau A_{2}\right) \mathbf{w}^{n}\right\|^{2} & =\left(\left(E+\tau A_{2}\right) \mathbf{w}^{n},\left(E+\tau A_{2}\right) \mathbf{w}^{n}\right) \\
& =\left(\mathbf{w}^{n}, \mathbf{w}^{n}\right)+2 \tau\left(A_{2} \mathbf{w}^{n}, \mathbf{w}^{n}\right)+\tau^{2}\left(A_{2} \mathbf{w}^{n}, A_{2} \mathbf{w}^{n}\right)
\end{aligned}
$$


and $A_{2}=P \geqslant 0$, we get

$$
\left\|\mathbf{w}^{n+1}\right\| \leqslant\left\|\left(E+\tau A_{2}\right) \mathbf{w}^{n+1}\right\| .
$$

From (37)-(39), we obtain the required a priory estimate of the discrete solution in the space $H_{*}^{2}$

$$
\left\|\mathbf{w}^{n+1}\right\| \leqslant\left(\left\|\mathbf{w}^{0}\right\|^{2}+\tau^{2}\left\|\operatorname{grad}_{h} p^{0}\right\|^{2}+2 \tau\left(\operatorname{grad}_{h} p^{0}, \mathbf{w}^{0}\right)\right)^{1 / 2}+\sum_{k=0}^{n} \tau\left\|\overline{\mathbf{f}}^{k}\right\|,
$$

where $\mathbf{w}^{0}=\mathbf{w}(\theta, 0), p^{0}=p(\theta, 0), \theta \in \omega$. Note that the estimate above is unconditional, i.e., it is obtained without any restrictions on the parameters of the computational grid.

\section{Numerical experiment}

A set of numerical experiments on the computation of the flow parameters in a cavity with the moving top wall has been performed using the proposed algorithm. We have considered nonstationary and stationary solutions. The criterion for obtaining a stationary solution is $\left\|\mathbf{w}_{\tau}\right\| \leqslant \varepsilon$, where $\varepsilon=10^{-5}$.

For the solution of the system of linear equations with a symmetric matrix determining the pressure correction and for linear problems with a nonsymmetric matrix determining the velocity field, the conjugate gradient method [11] and the modified strongly implicit method [13] have been used respectively.

We have compared the obtained steady-state results of the numerical experiments with the known benchmark solutions of an incompressible fluid flow in a cavity of a square section $[5,15]$ and a skewed one [12] with the skew angles $\alpha=30,45^{\circ}$.

Table 1 compares the steady-state results of the computation of the maximum values of the stream function $|\psi|_{\text {max }}$ in the square cavity for the Reynolds number Re=1000 obtained by different methods. Similar data for the case of a skewed cavity are presented in Tab. 2 and 3. The differences between the results of the benchmark computations can be due to the presence of the regularization term in the incompressibility condition affecting the stationary solution.

Table 1. Comparison of benchmark computations for the square cavity

\begin{tabular}{|r|r|r|r|}
\hline $\operatorname{Re}$ & {$[5], 257 \times 257$} & {$[15], 161 \times 161$} & $161 \times 161$ \\
\hline 100 & 0.1034 & 0.1000 & 0.1020 \\
\hline 400 & 0.1139 & 0.1069 & 0.1090 \\
\hline 1000 & 0.1179 & 0.1087 & 0.1105 \\
\hline 3200 & 0.1204 & 0.1003 & 0.1064 \\
\hline
\end{tabular}


Table 2. Comparison of benchmark computations for the skewed cavity with the skew angle $\alpha=30^{\circ}$

\begin{tabular}{|c|c|c|c|c|}
\hline \multirow{2}{*}{$\alpha=30^{\circ}$} & \multicolumn{2}{|c|}{$\operatorname{Re}=100$} & \multicolumn{2}{c|}{$\mathrm{Re}=1000$} \\
& $\min$ & $\max$ & $\min$ & $\max$ \\
\hline $161 \times 161$ & & & & \\
$\psi$ & $-5.3013 \mathrm{E}-02$ & $3.8321 \mathrm{E}-05$ & $-3.7887 \mathrm{E}-02$ & $3.3457 \mathrm{E}-03$ \\
$\mathrm{x}$ & 1.1674 & 0.5131 & 1.4582 & 0.8963 \\
$\mathrm{y}$ & 0.3781 & 0.1375 & 0.4125 & 0.2469 \\
\hline$[12], 320 \times 320$ & & & & \\
$\psi$ & $-5.3004 \mathrm{E}-02$ & $5.7000 \mathrm{E}-05$ & $-3.8185 \mathrm{E}-02$ & $3.8891 \mathrm{E}-03$ \\
$\mathrm{x}$ & 1.1674 & 0.5211 & 1.4583 & 0.8901 \\
$\mathrm{y}$ & 0.3781 & 0.1543 & 0.4109 & 0.2645 \\
\hline
\end{tabular}

Table 3. Comparison of benchmark computations for the skewed cavity with the skew angle $\alpha=45^{\circ}$

\begin{tabular}{|c|c|c|c|c|}
\hline \multirow{2}{*}{$\alpha=45^{\circ}$} & \multicolumn{2}{|c|}{$\mathrm{Re}=100$} & \multicolumn{2}{c|}{$\mathrm{Re}=1000$} \\
& $\min$ & $\max$ & $\min$ & $\max$ \\
\hline $161 \times 161$ & & & & \\
$\psi$ & $-6.9866 \mathrm{E}-02$ & $1.9883 \mathrm{E}-05$ & $-5.1805 \mathrm{E}-02$ & $8.5907 \mathrm{E}-03$ \\
$\mathrm{x}$ & 1.1128 & 0.3251 & 1.3144 & 0.7762 \\
$\mathrm{y}$ & 0.5503 & 0.1376 & 0.5769 & 0.3950 \\
\hline$[12], 320 \times 320$ & & & & \\
$\psi$ & $-7.0129 \mathrm{E}-02$ & $3.9227 \mathrm{E}-05$ & $-5.2553 \mathrm{E}-02$ & $1.0039 \mathrm{E}-02$ \\
$\mathrm{x}$ & 1.1146 & 0.3208 & 1.3120 & 0.7766 \\
$\mathrm{y}$ & 0.5458 & 0.1989 & 0.5745 & 0.3985 \\
\hline
\end{tabular}

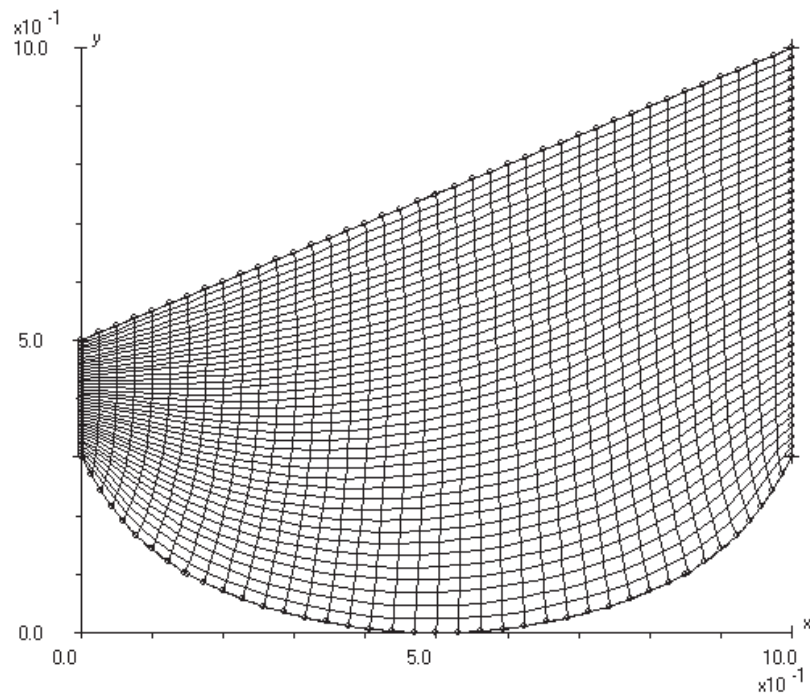

Figure 1. The $41 \times 41$ difference grid in the physical region $\Omega$ 

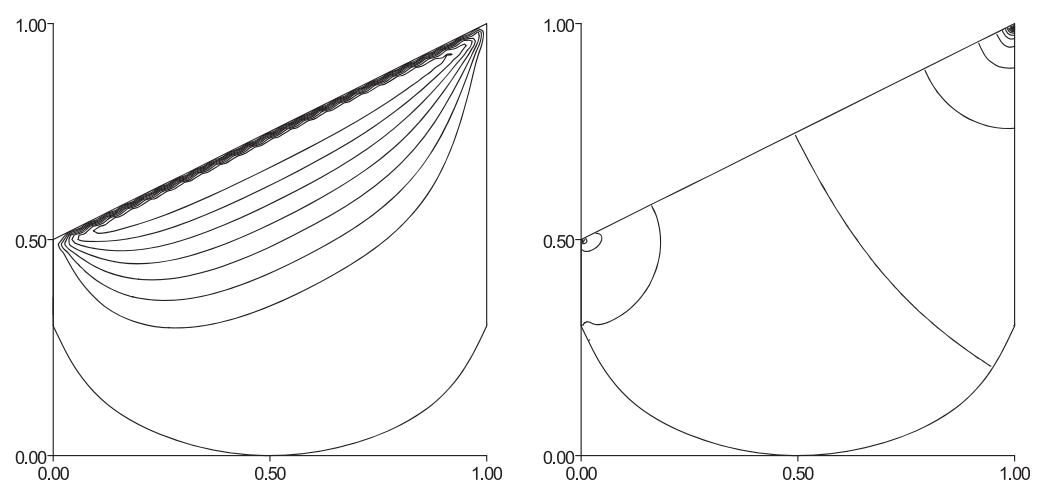

(a)
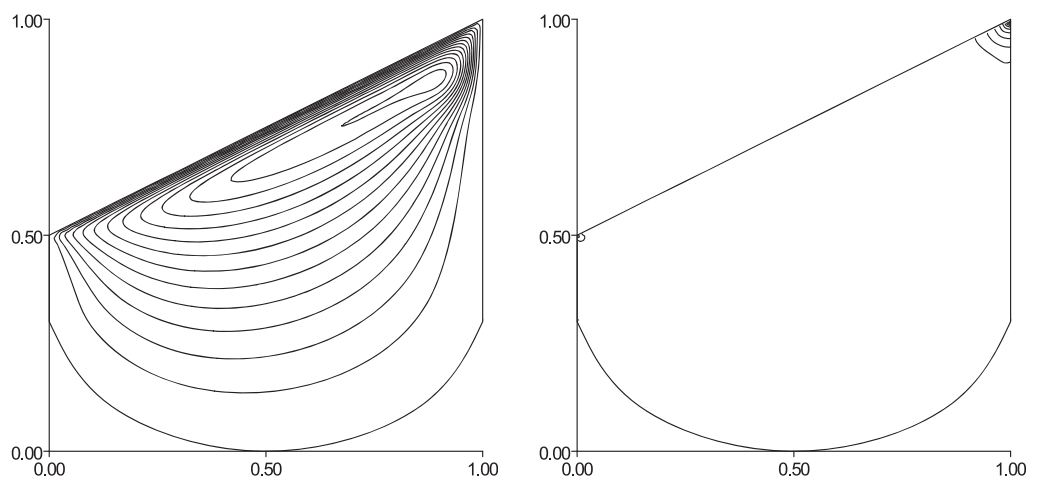

(b)
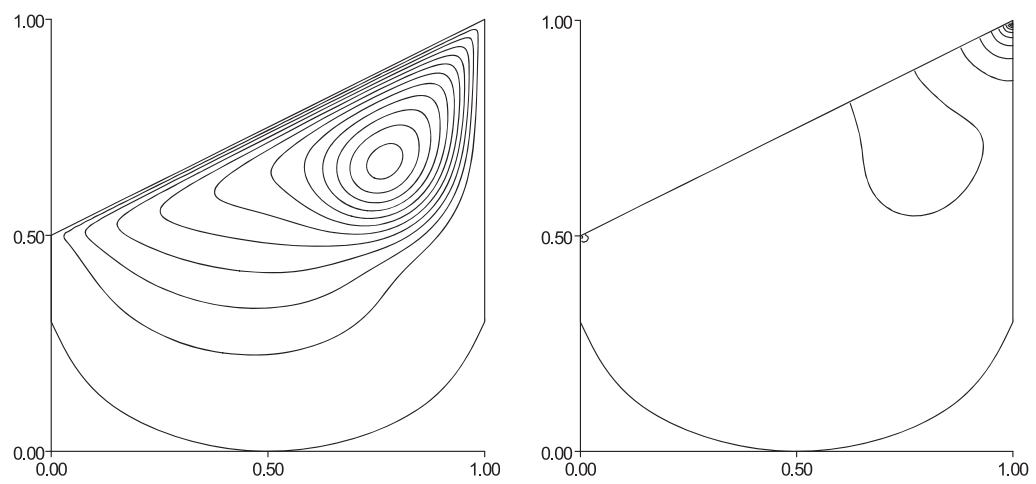

(c)

Figure 2. Stream lines and isobars of the nonstationary solution, $\operatorname{Re}=1000$, the $161 \times 161$ grid; (a) $t=0.1 ; \psi_{\max }=5.7967 E-05, \psi_{\min }=-9.0000 E-03, p_{\max }-p_{\min }=1.4936 ;(\mathrm{b})-t=1.0 ; \psi_{\max }=$ $3.9052 E-05, \psi_{\min }=-2.7047 E-02, p_{\max }-p_{\min }=1.7531 ;(\mathrm{c})-t=4.0 ; \psi_{\max }=1.1828 E-04$, $\psi_{\min }=-5.7084 E-02, p_{\max }-p_{\min }=1.8203$

We have performed the numerical experiment to model a nonstationary incompressible fluid flow in a driven cavity of arbitrary shape. The transformation of the source region into a parametrical square is done using the elliptical generator of difference grids [14]. Fig. 1 gives the $41 \times 41$ difference grid in the physical region $\Omega$.

The boundary value problem in the computational region $\Omega_{\xi \eta}$ for the system of equations (7)-(10) has been solved with the homogeneous initial condition $\mathbf{v}(\theta, 0)=0$ and the following boundary conditions

$$
\left.\mathbf{v}(\theta, t)\right|_{\theta \in \partial \Omega_{\xi \eta}}=\left\{\begin{array}{cc}
(\cos \beta, \sin \beta), & \eta=1 \\
(0,0), & \eta \neq 1
\end{array}\right.
$$


where $\beta$ is the skew angle of the moving top wall with the $O X$ axis. The computational results (stream lines and isobars) for the nonstationary problem are presented in Fig. 2.

The results of the computations of the stationary (steady-state) solutions for the various Reynolds numbers Re=100, 1000, 3200 on a uniform $161 \times 161$ grid are given in Figs. 3-5.
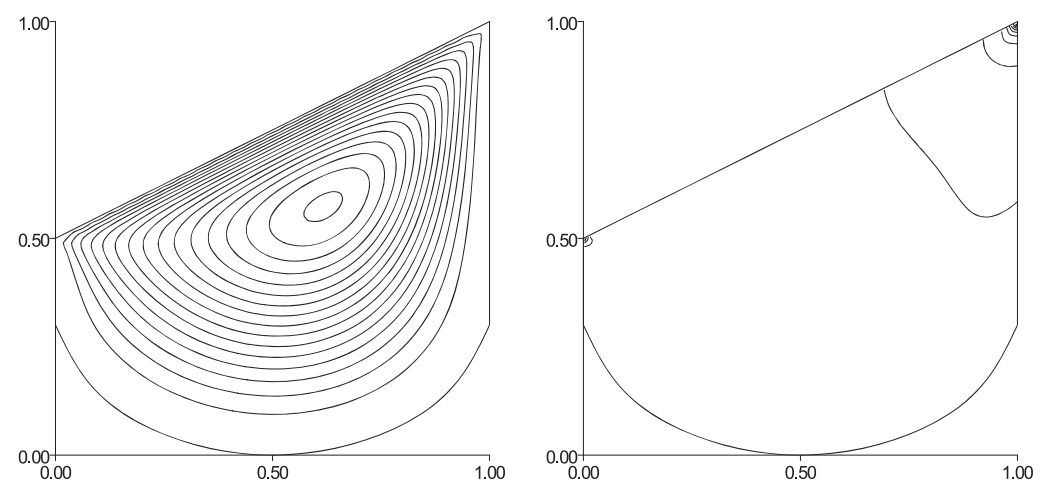

Figure 3. Stream lines and isobars of the stationary solution, $R e=100$, the $161 \times 161$ grid; $\psi_{\max }=$ $6.9046 E-05, \psi_{\min }=-8.0924 E-02, p_{\max }-p_{\min }=8.0714$
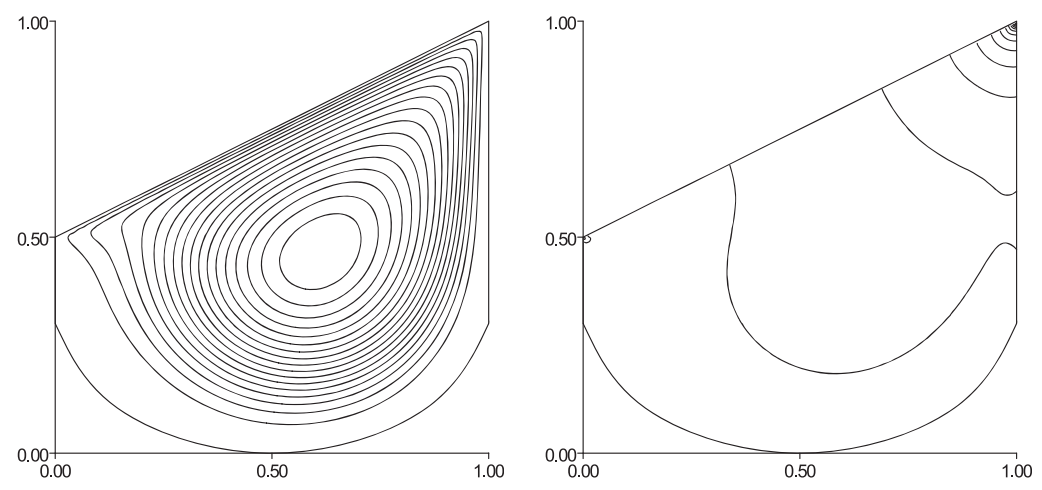

Figure 4. Stream lines and isobars of the stationary solution, $R e=1000$, the $161 \times 161$ grid; $\psi_{\max }=$ $3,4131 E-05, \psi_{\min }=-8,9705 E-02, p_{\max }-p_{\min }=1,8545$
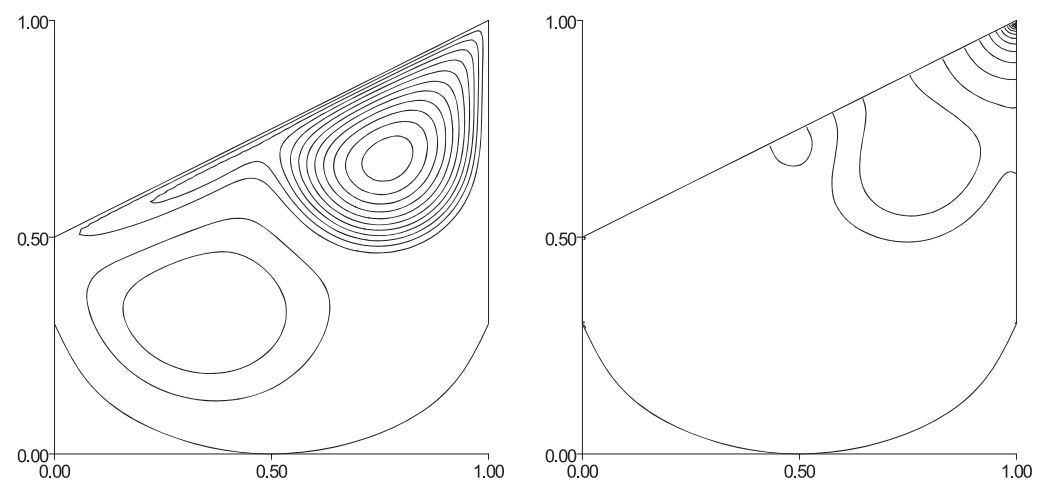

Figure 5. Stream lines and isobars of the stationary solution, $\operatorname{Re}=3200$, the $161 \times 161$ grid; $\psi_{\max }=$ $1.4696 E-02, \psi_{\min }=-5.8224 E-02, p_{\max }-p_{\min }=1.0799$

Figure 6 shows the value $|\psi|_{\max }$ versus time for different time steps $\tau$ on the $161 \times 161$ grid. A good accuracy of the solution of the nonstationary problem is achieved at $\tau \leqslant 0.25$. 
In practice the value of $\tau$ is chosen from the solution accuracy demands and does not affect the stability of the algorithm.

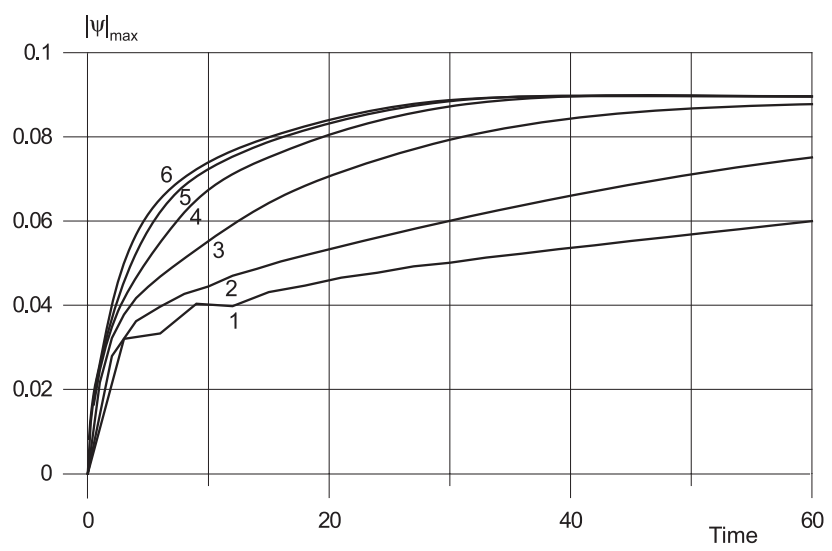

Figure 6. The value $|\psi|_{\max }$ versus time for different $\tau$, $\operatorname{Re}=1000$, the $161 \times 161$ grid; $1-\tau=3 ; 2-$ $\tau=2 ; 3-\tau=1 ; 4-\tau=0.5 ; 5-\tau=0.25 ; 6-\tau=0.1$

Figure 7 presents the value $|\psi|_{\max }$ versus time for different grids in the computational space and a small time step $\tau=0.02$.

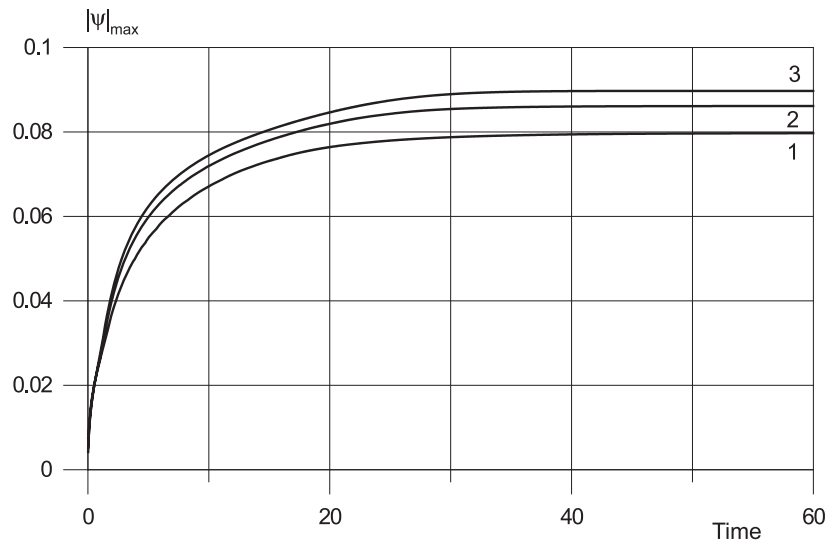

Figure 7. The value $|\psi|_{\max }$ versus time for different grids, $\operatorname{Re}=1000, \tau=0.02 ; 1-41 \times 41 ; 2-81 \times 81$; $3-161 \times 161$

\section{Conclusions}

The computational algorithm for the solution of the Navier-Stokes equations in natural variables on a nonstaggered grid in an arbitrary region is proposed. The second order approximations in the grid nodes and the Douglas-Rachford splitting method have been used in implementing the algorithm. The convective difference operator is skew-symmetric and the diffusion difference operator is self-adjoint and positive definite. The a priory estimate for the discrete solution has been obtained.

The regularization term for the elimination of oscillations of the discrete solution arising on nonstaggered grids has been added to the incompressibility condition and its properties have been examined. The use of the regularization term does not make the conservativeness 
of the difference scheme worse and does not affect the a priory estimate of the discrete solution. At the same time the algorithm is still easy to implement.

The proposed algorithm has been checked on a series of test problems and its efficiency for obtaining of stationary and nonstationary solutions has been proved. The first order of approximation of the incompressibility condition at boundary nodes in the computational space is the chief disadvantage of the scheme.

\section{Acknowledgements}

The authors wish to thank Prof. Piotr Vabischevich and Prof. Piotr Matus for the discussion and usefull hints.

\section{References}

[1] S. Acharia and F. Moukalled, Improvements to incompressible flow calculation on a non-staggered curvilinear grid, Numer. Heat Transfer, 15 (1989), pp. 131-152.

[2] I. Demirdzic, Z. Lilek, and M. Peric, Fluid flow and heat transfer test problems for non-orthogonal grids: Bench-mark solutions, Int. J. Numer. Meth. Fluids, 15 (1992), pp. 329-354.

[3] I. Demirdzic and M. Peric, Finite volume method for prediction of fluid flow in arbitrary shaped domains with moving boundaries, Int. J. Numer. Meth. Fluids, 10 (1990), pp. 771-790.

[4] J. Douglas and J. Gunn, A general formulation of alternating direction methods. Part 1. Parabolic and hyperbolic problems, Numer. Math., 6 (1964), no. 5.

[5] U. Ghia, K. Ghia, and C. Shin, High-Re solutions for incompressible flow using the Navier-Stokes equations and a multigrid method, J. Comput. Phys., 48 (1982), pp. $387-411$.

[6] S. Koshizuka, Y. Oka, and S. Kondo, A staggered differencing technique on boundary-fitted curvilinear grids for incompressible flows along curvilinear or slant walls, Comput. Mech., 7 (1990), pp. 123 - 136.

[7] C. Oosterlee, P. Wesseling, A. Segal, and E. Brakkee, Benchmark solutions for the incompressible Navier-Stokes equations in general coordinates on staggered grids, Int. J. Numer. Meth. Fluids, 17 (1993), pp. 301-321.

[8] M. Rosenfeld, D. Kwak, and M. Vinokur, A fractional step solution method for the unsteady incompressible Navier-Stokes equations in generalized coordinate systems, J. Comput. Phys., 94 (1991), pp. $102-137$.

[9] A. Samarskii, The Theory of Difference Schemes, Marcel Dekker Inc., New York-Basel, 2001.

[10] A. Samarskii and V. Andreev, Difference Methods for Elliptic Equations, Nauka, Moskow, 1976.

[11] A. Samarskii and P. Vabishchevich, Computational Heat Transfer, vol. 1 and 2, Wiley, Chichester, 1995.

[12] A. Shklyar and A. Arbel, Numerical method for calculation of the incompressible flow in general curvilinear coordinates with double staggered grid, Int. J. Numer. Meth. Fluids, 41 (2003), no. 12, pp. 1273 $-1294$.

[13] G. Shneider and M. Zedan, A modified strongly implicit procedure for the numerical solution of field problems, Numer. Heat Transfer, 4 (1981), no. 1, pp. 1 - 19.

[14] J. Thompson, Z. Warsi, and C. Mastin, Boundary fitted coordinate systems for numerical solution of partial differential equations. - a review, J. Comput. Phys., 47 (1982), no. 1, pp. 1 - 108.

[15] P. Vabishchevich, A. Pavlov, and A. Churbanov, Numerical methods for unsteady incompressible flows using primitive variables and non-staggered grids, Matematicheskoe modelirovanie, 8 (1996), no. 7, pp. $81-108$. 
[16] P. Wesseling, A. Segal, J. van Kan, C. Oosterlee, and C. Kassels, Finite volume discretization of the incompressible Navier-Stokes equations in general coordinates on staggered grids, Comput. Fluids Dyn. Journal, 1 (1992), pp. 27 - 33.

[17] Y. Zang, R. L. Street, and J. R. Koseff, A non-staggered grid, fractional step method for time-dependent incompressible Navier-Stokes equations in curvilinear coordinates, J. Comput. Phys., 114 (1994), pp. $18-33$. 